\title{
PABPN1 shuts down alternative poly(A) sites
}

\author{
Martine Simonelig ${ }^{1}$ \\ ${ }^{I} m R N A$ Regulation and Development, Institute of Human Genetics, CNRS UPR1142, 141 rue de la Cardonille, 34396 Montpellier \\ Cedex 5, France \\ Cell Research (2012) 22:1419-1421. doi:10.1038/cr.2012.86; published online 29 May 2012
}

\begin{abstract}
Although overlooked for many years, alternative cleavage and polyadenylation (APA) is now emerging as a major mechanism of gene regulation. A recent study identifies poly(A)binding protein nuclear 1 (PABPN1), a general factor of polyadenylation, as a suppressor of alternative poly(A) sites.
\end{abstract}

mRNA 3 '-end processing is a cotranscriptional reaction that leads to the addition of a poly(A) tail - polyadenylation - to virtually all eukaryotic mRNAs. The reaction occurs in two steps: the endonucleolytic cleavage of the pre-mRNA at the poly(A) site and the synthesis of a poly(A) tail onto the upstream cleaved molecule [1]. The poly(A) tail has essential functions in many aspects of the life of mRNAs including export to the cytoplasm, stability, intracellular localization and translation. mRNA 3'-end processing requires four multiprotein cleavage factors (CPSF: cleavage and polyadenylation specificity factor; CstF: cleavage stimulation factor; CF I and II: cleavage factors I and II), additional accessory proteins such as RNA polymerase II and symplekin, the poly(A) polymerase (PAP) that synthesizes the poly(A) tail and the poly(A)-binding protein nuclear 1 (PABPN1). Poly(A) sites are defined

Correspondence: Martine Simonelig

Tel: +33 43435 99 59; Fax: +33434359957

E-mail: Martine.Simonelig@igh.cnrs.fr by two sequences, the canonical poly(A) signal AAUAAA localized upstream of the cleavage site and a downstream U/GU-rich motif; both motifs cooperatively recruit the polyadenylation machinery through direct interactions with CPSF and CstF, respectively.

Recent studies using genome-wide approaches have revealed alternative cleavage and polyadenylation (APA) to be very widespread. More than $50 \%$ of human genes generate multiple transcripts with different $3^{\prime}$ UTRs resulting from APA. Alternative poly(A) site usage can lead to the production of different protein isoforms, or the production of different transcripts encoding the same protein with $3^{\prime}$ UTRs of varying lengths. Because the 3' UTRs represent binding platforms for RNA-binding proteins and microRNAs, changes in the length of 3' UTRs can strongly affect the cytoplasmic regulation of mRNAs including their stability, localization and translation rate, with longer 3' UTRs being more likely to suffer a negative regulation [2]. Genome-wide analyses have revealed global shortening of 3' UTRs in proliferating cells, undifferentiated cells including induced pluripotent stem cells reprogrammed from somatic cells, and cancer cell lines, whereas 3' UTRs were found to lengthen during embryonic development and cell differentiation [2]. A recent study in Drosophila showed a general trend for very long 3' UTRs (up to $18 \mathrm{~kb}$ ) in the central nervous system [3].
It has been known for many years that, in addition to specific regulators of APA, an important mechanism underlying APA involves changes in the concentration of general cleavage and polyadenylation factors, CstF being the first complex to be implicated in this regulation [4-7]. From these analyses, a general view emerged that promoter-proximal poly(A) sites tend to be weaker than distal poly(A) sites, and that increased levels of the core cleavage and polyadenylation machinery favor weak proximal sites (Figure 1). Consistent with this, short $3^{\prime}$ UTRs in undifferentiated and cancer cells correlate with upregulation of cleavage and polyadenylation factors, including CPSF, CstF and symplekin, whereas the lengthening of 3' UTRs in differentiated cells correlates with the downregulation of the same factors [2].

The recent study by Jenal et al. [8] now showed that PABPN1 plays a role in APA. Strikingly, in contrast to other cleavage and polyadenylation factors, PABPN1 prevents the usage of proximal poly(A) sites.

PABPN1 was first identified for its role during the second step of the cleavage and polyadenylation reaction, polyadenylation. In vitro experiments have shown that PABPN1 has two functions during polyadenylation: it firstly binds nascent poly(A) tails and stimulates PAP together with CPSF by stabilizing the interaction of PAP with RNA [9], then it stops elongation when the poly(A) tail 
has reached a length of 250 nucleotides by disrupting PAP-CPSF association [10]. A role in poly(A) tail lengthening has been validated in vivo using mutations in the Drosophila homologue of PABPN1 (Pabp2). Analysis of these mutants revealed an additional function of PABP2/PABPN1 in cytoplasmic mRNA poly(A) tail length regulation during early development [11]. Recent studies in $S$. pombe identified even broader functions of the PABPN1 homologue via its interaction with the nuclear exosome, in the maturation of non-coding small nucleolar RNAs and in the nuclear degradation of unspliced pre-mRNAs $[12,13]$.

Adding to the already diverse functional repertoire of PABPN1 in RNA metabolism, Jenal et al. [8] identified yet another novel function for this protein. Using a RNAi screen for RNAbinding proteins in $\mathrm{U} 2 \mathrm{OS}$ cells to identify regulators of APA, PABPN1 was found to be the top-scoring candidate. Decreased amounts of PABPN1 favored usage of a proximal poly(A) site. 3 ' end deep-sequencing was applied to address the generality of this effect. Of a little over 9000 sequenced transcripts, $32 \%$ had more than one cleavage site in their 3' UTR. A decrease in PABPN1 levels resulted in a shift in poly(A) site usage for 572 transcripts, with increased usage of the proximal site in most cases (91\%), thus revealing extensive $3^{\prime}$ UTR shortening upon PABPN1 reduction. Proximal poly(A) sites are generally weaker due to non-canonical poly(A) signals and accordingly variants to the canonical AAUAAA poly(A) signal were enriched in proximal poly(A) site regions. These results suggested that reduction in PABPN1 enhanced usage of non-canonical proximal poly(A) sites and therefore that PABPN1 might actively prevent their usage. Addressing the mechanism behind this regulation, the authors went on to show that PABPN1 could indeed be crosslinked to proximal poly(A) site regions, indicating direct interactions of PABPN1 with these regions. Binding of PABPN1 to proximal poly(A) site regions was confirmed in vitro and depended on the integrity of the non-canonical poly(A) signal. In addition, enhanced cleavage at proximal poly(A) sites upon reduction of PABPN1 was recapitulated in in vitro cleavage assays. Importantly, this study also showed using cyclin D1 mRNA as a model, that increased proximal poly(A) site usage upon reduction of PABPN1 prevented mRNA regulation by microRNAs targeting the $3^{\prime}$ UTR downstream of the proximal poly(A) site.

Based on these results, the authors proposed that PABPN1 plays an active role in the repression of proximal poly(A) site usage by direct binding to non-canonical poly(A) signals; PABPN1 binding would compete with the recruitment of CPSF on these weak poly(A) signals. Not only does this study identify a new function of PABPN1 in APA, prior to cleavage, but it also changes the current view of regulation of poly(A) site usage by general
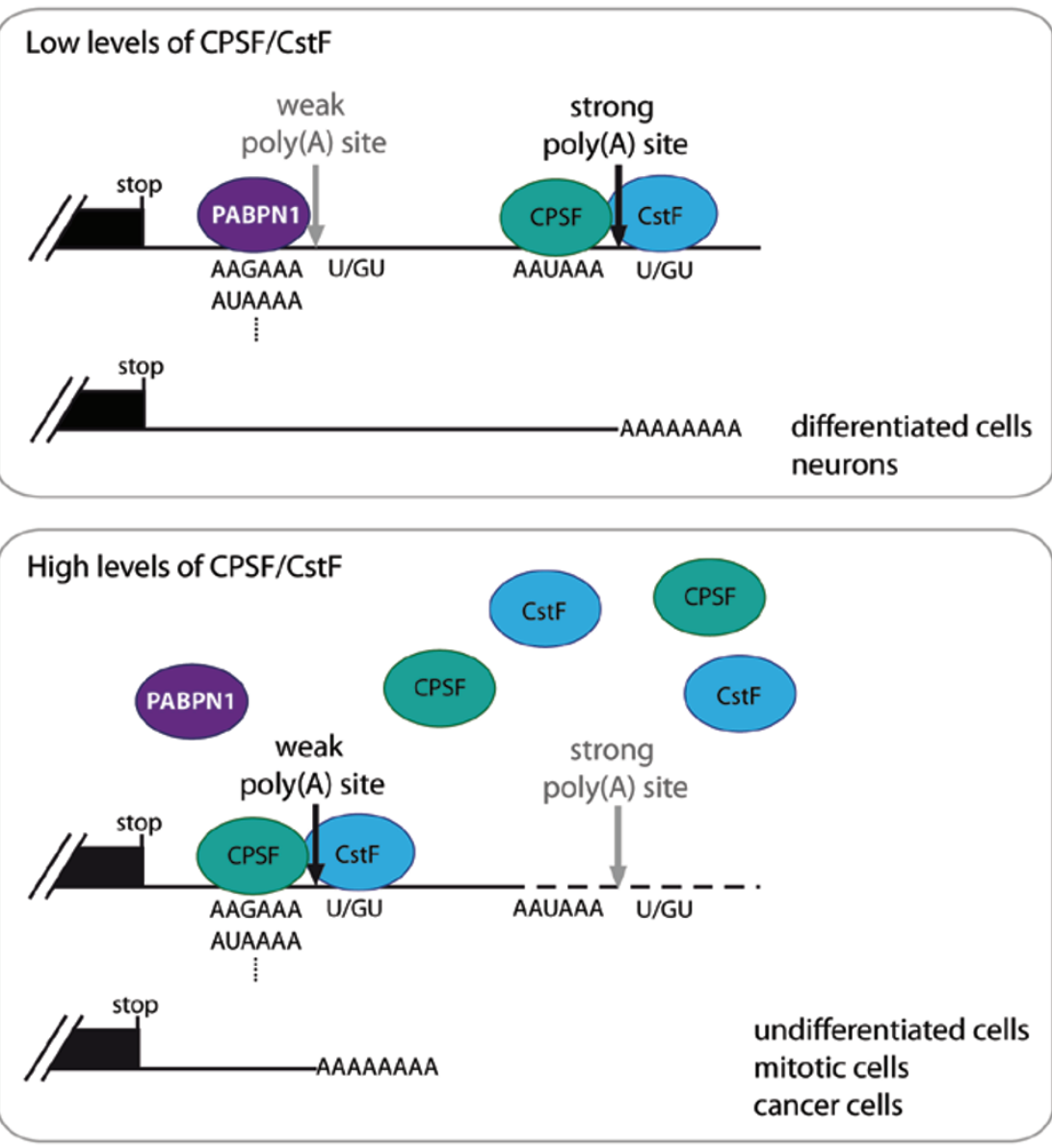

Figure 1 Proposed model for the regulation of APA integrating the role of PABPN1 and of CPSF and CstF levels. Top panel: Low levels of CPSF/CstF favor usage of strong poly $(A)$ sites with canonical poly $(A)$ signals; PABPN1 bound to non-canonical poly $(A)$ signals competes with binding by CPSF and actively prevents usage of weak proximal poly $(A)$ sites. Bottom panel: High levels of CPSF/CstF, or reduction of PABPN1 such as is observed in OPMD, favor usage of weak proximal poly $(\mathrm{A})$ sites through the binding of CPSF/CstF to non-canonical motifs. The dotted line indicates that cleavage at the proximal poly $(A)$ site might occur before transcription of the distal poly $(A)$ site. 
cleavage and polyadenylation factors, suggesting that the low affinity of these factors for non-canonical motifs in weak poly(A) sites might not be sufficient to prevent their usage and that an active role of PABPN1 is also required (Figure 1). Binding of PABPN1 to canonical poly(A) signals in strong poly(A) sites would not affect usage of these sites due to the enhanced recruitment of CPSF and CstF to canonical motifs.

It would now be of interest to address the contribution of PABPN1 in APA in physiological or pathological conditions such as differentiation or cancer where global shifts in poly(A) site usage correlate with changes in CPSF and CstF levels.

Interestingly, dominant mutations corresponding to short polyalanine expansions in PABPN1 lead to a genetic disorder called oculopharyngeal muscular dystrophy (OPMD) [14]. Using cell and mouse models of OPMD, Jenal et al. [8] found a global shift towards proximal poly(A) site usage in these models, correlating with an upregulation of transcripts with short 3' UTRs. PABPN1 is known to self-interact to carry out its function in polyadenylation, and alanine-expanded PABPN1 was found to interact with normal PABPN1. This led to the suggestion that, in OPMD, normal PABPN1 might be sequestered by alanine-expanded PABPN1 into nuclear inclusions, a hallmark of the disease, thus resulting in depletion of the normal protein. These results highlight the major role of PABPN1 in APA and provide an example in a pathological condition where changes in PABPN1 levels result in a shift in poly(A) site usage. They also suggest a potential important role of APA in OPMD. How APA indeed contributes to OPMD pathology is now a very interesting question to address.

\section{Acknowledgments}

I thank Aymeric Chartier, Isabelle Busseau and Elmar Wahle for discussions. Work in the Simonelig lab is supported by the CNRS UPR1142, ANR (ANR-2010-BLAN-1201 01 and ANR-2009-GENO-025-01), FRM ("Equipe FRM 2007" and "Projets Innovants ING20101221078"), ARC (ARC Libre 2009 N³192) and AFM (Research Program 15123).

\section{References}

1 Proudfoot NJ. Ending the message: poly(A) signals then and now. Genes Dev 2011; 25:1770-1782.

2 Di Giammartino DC, Nishida K, Manley JL. Mechanisms and consequences of alternative polyadenylation. Mol Cell 2011; 43:853-866.

3 Smibert P, Miura P, Westholm JO, et al. Global patterns of tissue-specific alternative polyadenylation in Drosophila. Cell Rep 2012; 1:277-289.

4 Audibert A, Simonelig M. Autoregulation at the level of mRNA 3'-end formation of the suppressor of forked gene of Drosophila melanogaster is conserved in Drosophila virilis. Proc Natl Acad Sci USA 1998; 95:14302-14307.

5 Juge F, Audibert A, Benoit B, Simonelig M. Tissue-specific autoregulation of Drosophila suppressor of forked by alternative poly(A) site utilization leads to accumulation of the suppressor of forked protein in mitotically active cells. RNA 2000; 6:1529-1538.

6 Takagaki Y, Manley JL. Levels of polyadenylation factor CstF-64 control IgM heavy chain mRNA accumulation and other events associated with B cell differentiation. Mol Cell 1998; 2:761-
771.

7 Takagaki Y, Seipelt RL, Peterson ML, Manley JL. The polyadenylation factor CstF-64 regulates alternative processing of IgM heavy chain pre-mRNA during B cell differentiation. Cell 1996; 87:941-952.

8 Jenal M, Elkon R, Loayza-Puch F, et $a l$. The poly(a)-binding protein nuclear 1 suppresses alternative cleavage and polyadenylation sites. Cell 2012; 149:538-553.

9 Kerwitz Y, Kuhn U, Lilie $\mathrm{H}$, et al. Stimulation of poly(A) polymerase through a direct interaction with the nuclear poly(A) binding protein allosterically regulated by RNA. EMBO $J$ 2003; 22:3705-3714.

10 Kuhn U, Gundel M, Knoth A, Kerwitz Y, Rudel S, Wahle E. Poly(A) tail length is controlled by the nuclear poly(A)-binding protein regulating the interaction between poly(A) polymerase and the cleavage and polyadenylation specificity factor. $J \mathrm{Biol}$ Chem 2009; 284:22803-22814.

11 Benoit B, Mitou G, Chartier A, et al. An essential cytoplasmic function for the nuclear poly(A) binding protein, PABP2, in poly(A) tail length control and early development in Drosophila. Dev Cell 2005; 9:511-522.

12 Lemay JF, D'Amours A, Lemieux C, et $a l$. The nuclear poly(A)-binding protein interacts with the exosome to promote synthesis of noncoding small nucleolar RNAs. Mol Cell 2010; 37:34-45.

13 Lemieux C, Marguerat S, Lafontaine J, Barbezier N, Bahler J, Bachand F. A Pre-mRNA degradation pathway that selectively targets intron-containing genes requires the nuclear poly(A)binding protein. Mol Cell 2011; 44:108119.

14 Brais B, Bouchard J-P, Xie Y-G, et al. Short GCG expansions in the PABP2 gene cause oculopharyngeal muscular dystrophy. Nat Genet 1998; 18:164167. 